\title{
A SIMPLE PROOF OF A COVERING PROPERTY OF LOCALLY COMPACT GROUPS
}

\author{
P. MILNES ${ }^{1}$ AND J. V. BONDAR
}

\begin{abstract}
We give a simple proof of the following result of Emerson and Greenleaf.

THEOREM. Let $V$ be a relatively compact subset with nonvoid interior of a locally compact group $G$. Then there exist a subset $T \subset G$ and a natural number $M$ such that $G=\bigcup_{t \in T} t V$ and at most $M$ of the $t V$ 's, $t \in T$, intersect.
\end{abstract}

The result cited above is proved in [4] and is used there and in [2] in the course of proving that every amenable locally compact group $G$ has strong properties such as

(A) If $\varepsilon>0$ and compact $K \subset G$ containing the identity of $G$ are given, there is a compact $U \subset G$ with $|U|>0$ such that $|K U \Delta U| /|U|<\varepsilon$.

(Here $|U|$ indicates left Haar measure of the set $U$. And we remind the reader that $G$ is called amenable if $L^{\infty}(G)$ admits a left invariant mean; see [6], [8], [1] for further details.)

The proof given in [4] of the theorem above involves some delicate arguments about geometry of groups. We discovered the simple proof presented below in the course of preparing [1] (and were apprised later that it is almost the same as a proof in Chapter 8, $\$ 1.7$, of [7]); our reason for publishing it now is that it seems not widely known, according to [3], [5], that such a proof exists.

Proof of THE THEOREM. After a reduction as in [2; Proposition 2], we are left with the task of taking a relatively compact symmetric neighbourhood $V$ of $e \in G$ and finding $T \subset G$ and constant $M$ so that $G=\cup_{t \in T^{t}} V$ and at most $M$ of the $t V$ 's, $t \in V$, intersect. We may assume the open and closed subgroup $\cup_{1}^{\infty} V^{n}$ of $G$ equals $G$. (For, if we cover $\cup_{1}^{\infty} V^{n}$ with $\cup_{t \in T} t V$, then $\cup_{t \in T} s t V$ covers the coset $s \cup{ }_{1}^{\infty} V^{n}$ and hence we cover the whole group.) And we may assume the subgroup $\cup_{1}^{\infty} V^{n}$ is not compact. (Otherwise we can cover it with a finite number of left translates of $V$ and proceed as in the previous parenthetical remark.) We then get our set $T \subset G$ as follows.

Let $t_{1}=e$. Since $G$ is not compact, $V^{2} \neq V$ and there is a $t_{2} \in\left(V^{2}\right)^{-} \backslash V$.

Received by the editors February 22, 1978 and, in revised form, April 28, 1978.

AMS (MOS) subject classifications (1970). Primary 22D05, $43 \mathrm{~A} 07$.

Key words and phrases. Locally compact group, amenability, covering property.

${ }^{1}$ The research of the first-named author was supported in part by NRC grant A7857.

$0002-9939 / 79 / 0000-0024 / \$ 01.50$

(C) 1979 American Mathematical Society 
If $\left(V^{2}\right)^{-} \backslash \cup_{1}^{2} t_{i} V \neq \varnothing$, take $t_{3}$ in it. Continuing like this, we get $\left(V^{2}\right)^{-} \subset$ $\cup_{1}^{N_{2}} t_{i} V$ with

$$
t_{j} \notin \bigcup_{1}^{j-1} t_{i} V, \quad 2 \leqslant j \leqslant N_{2} .
$$

(Note that, if $W$ is a symmetric neighbourhood of $e$ such that $W^{2} \subset V$, then

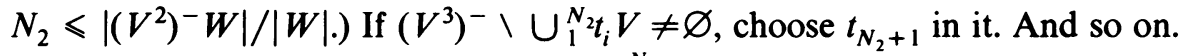
Hence, by induction, we get $\left(V^{n}\right)^{-} \subset \cup_{1}^{N^{n} t_{i} V \text { with }}$

$$
t_{j} \notin \bigcup_{1}^{j-1} t_{i} V, \quad 2 \leqslant j \leqslant N_{n} ;
$$

thus $G=\cup_{1}^{\infty} t_{i} V$ (and $N_{n} \leqslant\left|\left(V^{n}\right)^{-} W\right| /|W|$ ). Suppose $s \in t_{i} V$. Then $t_{i} \in s V$ and $t_{i} W \in s V W$ (where $W^{2} \subset V$ as above). And, if $s$ is also in $t_{j} V$, then $t_{j} W \subset s V W$ with $t_{i} W \cap t_{j} W=\varnothing$ if $i \neq j$. It follows that $s$ is contained in at most $|V W| /|W|$ of the $t_{i} V$ 's, $i=1,2,3, \ldots$

\section{REFERENCES}

1. J. V. Bondar and P. Milnes, A survey of Hunt-Stein and related conditions on groups (submitted).

2. W. R. Emerson, Ratio properties in locally compact groups, Trans. Amer. Math. Soc. 133 (1968), 179-204.

3. __ Letter to the first-named author, January 1978.

4. W. R. Emerson and F. P. Greenleaf, Covering properties and Folner conditions for locally compact groups, Math. Z. 102 (1967), 370-384.

5. P. Eymard, Initiation à la théorie des groupes moyennables, Analyse Harmonique sur les Groupes de Lie, Eymard et al., eds., Lecture Notes in Math., vol. 497, Springer-Verlag, Berlin, 1975, pp. 89-107.

6. F. P. Greenleaf, Invariant means on topological groups, Van Nostrand Math. Studies Series, no. 16, Van Nostrand, New York, 1969.

7. H. Reiter, Classical harmonic analysis and locally compact groups, Oxford Univ. Press, Cambridge, 1968.

8. M. Stone and R. von Randow, Statistically inspired conditions on the group structure of invariant experiments ..., Z. Wahrscheinlichkeitstheorie und Verw. Gebiete 10 (1968), 70-80.

Department of Mathematics, University of Western Ontario, London, Ontario N6a 3K7, CANADA

Research Branch, Canadian Radio-Television Commission, Ottawa, Ontario K1A 0N2, Canada 